Allton Augustinho MarCHI

Maria Salete Costa Gurget ${ }^{2}$

Artigo original

Palavras-chave

Neoplasias da mama Mamografia

Programas de rastreamento Implementação de planos de saúde

Fidelidade a diretrizes

Keywords

Breast neoplasms Mammography

Mass screening

Health plan implementation Guideline adherence

\section{Adesão ao rastreamento mamográfico oportunístico em serviços de saúde públicos e privados}

\author{
Adherence to the opportunistic mammography screening in public and \\ private bealth systems
}

l

\title{
Resumo
}

OBJETIVO: avaliar a adesão às recomendações para o rastreamento mamográfico oportunístico do câncer de mama. MÉTODOS: estudo prospectivo que acompanhou 460 mulheres na cidade de Taubaté, São Paulo, Brasil, das quais 327 foram atendidas em serviços de saúde públicos e 133 em Serviços privados, durante período de cinco anos após a realização de mamografia índice. Analisamos a prevalência de repetição da mamografia, as taxas de adesão e os fatores preditivos associados ao rastreamento mamográfico vigente. As associações dos desfechos com as variáveis independentes foram estudadas pela obtenção dos riscos relativos (RR) e respectivos intervalos de confiança de 95\% (IC95\%). O cálculo das razões de prevalência ajustadas foi realizado pela técnica de regressão de COX. RESULTADOS: apesar de mais de 90\% das entrevistadas terem repetido pelo menos uma vez o exame de mamografia, a adesão correta às recomendações do rastreamento mamográfico, com a repetição do procedimento a cada 24 meses, revelou taxas muito baixas (ao redor de 30\%) na amostra estudada. Os fatores preditivos associados à adesão ao rastreamento mamográfico relacionaram-se com o acesso diferenciado aos serviços de saúde, públicos ou privados ( $R R=1,77 ; \mathrm{IC} 95 \%=1,26-2,48$ ) e com a presença de rastreio prévio (RR=3,07; IC95\%=1,86-5,08). CONCLUSÕES: ocorreu baixa adesão às recomendações do rastreamento mamográfico oportunístico do câncer de mama em ambos os segmentos populacionais estudados.

\section{Abstract}

PURPOSE: to assess compliance with the recommendations for opportunistic breast cancer screening by mammography. METHODS: 460 women from the town of Taubaté, São Paulo, Brazil, were followed-up prospectively after the index mammography, 327 of them attended by the public health system and 133 by the private system. We evaluated the prevalence of mammography repetition, the adherence rates and predictive factors associated with the current recommendations of mammographic screening. The association of the outcomes with the independent variables was studied by obtaining the risk rates (RR) and the respective $95 \%$ confidence intervals (95\% CI). The adjusted prevalence rates were calculated by the COX regression model. RESULTS: although more than $90 \%$ of the studied women repeated the mammography at least once, the rate of correct compliance with the recommendations of mammographic screening, with repetition of the procedure every 24 months, was low (about $30 \%$ of the study sample). The preditive factors associated with compliance with mammographic screening were related to the unequal access to public or private healthcare services $(\mathrm{RR}=1.77 ; 95 \% \mathrm{Cl}=1.26-2.48)$ and to previous screening ( $\mathrm{RR}=3.07 ; 95 \% \mathrm{Cl}=1.86-5.08)$. CONCLUSION: we concluded that compliance with the recommendations of opportunistic mammographic screening for breast cancer was low in both studied population segments.

Correspondência:

Ailton Augustinho Marchi Serviço de Obstetrícia e Ginecologia do Hospital Universitário de Taubaté Avenida Granadeiro Guimarães, 270 - Centro (EP 12020-130 - Taubaté (SP), Brasil Fax: (12) 3622-8181 E-mail: aamarchi@uol.com.br
Serviço de Obstetrícia e Ginecologia do Hospital Universitário de Taubaté - Taubaté (SP), Brasil.

Professor Assistente do Departamento de Ginecologia e Obstetrícia da Faculdade de Medicina da Universidade de Taubaté UNITAU - Taubaté (SP), Brasil.

2 Professora Associada do Departamento de Tocoginecologia da Faculdade de Ciências Médicas da Universidade Estadual de Campinas - UNICAMP - Campinas (SP), Brasil. 


\section{Introdução}

O câncer de mama é a neoplasia mais frequente tanto entre as mulheres norte-americanas quanto entre as brasileiras $^{1,2}$. Estimou-se que, em 2009, de todos os tumores detectados na população feminina dos Estados Unidos, 27\% (194.200 novos casos) tiveram como sítio primário a glândula mamária ${ }^{1}$. No Brasil, as estimativas válidas para o biênio 2010/2011 apontam que ocorrerão 49.240 casos novos de câncer de mama anualmente, com risco estimado de 49 casos para cada 100 mil mulheres ${ }^{2}$.

A redução da mortalidade devida ao câncer entre as mulheres norte-americanas, no período de 2001 a 2005, foi da ordem de 11 pontos percentuais. Mais de um terço do decréscimo registrado deveu-se à redução da mortalidade correspondente ao tumor mamário. Esse declínio foi atribuído à utilização de terapêuticas mais eficazes associadas ao rastreamento e detecção precoce da doença ${ }^{1}$. Diversamente no Brasil, notou-se aumento na taxa de mortalidade ocasionada por câncer mamário nas últimas três décadas. Dados atuais, obtidos por meio do Instituto Nacional do Câncer (INCA), estimam a ocorrência de 15,6 óbitos por 100.000 mulheres/ano ${ }^{3}$.

A extensão inicial da doença é o principal preditor da sobrevida das mulheres acometidas por essa doença ${ }^{4}$. Muito embora análises temporais recentes das tendências do estadiamento do câncer de mama no Brasil indiquem maiores taxas de detecção precoce do tumor, um terço dos registros de casos novos ainda corresponde à doença localmente avançada ou tardiamente diagnosticada $a^{5-7}$.

Embora existam constantes questionamentos quanto ao papel da mamografia, estima-se decréscimo de $30 \%$ na mortalidade decorrente do câncer mamário em mulheres com idade acima de 50 anos rastreadas regularmente a cada 24 meses $^{8,9}$. O United States Preventive Task Force (USPTF) alterou recentemente suas recomendações para o início do rastreamento mamográfico do câncer de mama. No entanto, o rastreamento anual a partir de 40 anos de idade ainda é proposto por várias entidades norte-americanas ${ }^{10,11}$.

No Brasil, as diretrizes também são conflitantes. Recomenda-se o rastreamento mamográfico bienal - para mulheres não pertencentes a grupos populacionais de risco - somente a partir de 50 anos de idade ${ }^{12}$. No setor suplementar de assistência à saúde, defende-se a triagem mamográfica anual a partir de 40 anos, bienalmente entre 50 e 69 anos e, de acordo com a expectativa de vida da mulher, após os 70 anos de idade ${ }^{13}$.

Os benefícios plenos dos programas de rastreamento tornam-se evidentes e mensuráveis, entre outros fatores, na dependência tanto de sua extensão temporal quanto da adesão da população alvo às suas recomendações. Foram estabelecidos os modelos de rastreamento mamográfico populacional organizado e oportunístico ${ }^{14}$. No rastreamento mamográfico populacional organizado existe convocação e vigilância das mulheres inscritas, além do cumprimento dos intervalos entre as etapas propostas. No modelo oportunístico, os indivíduos somente são submetidos às recomendações a partir da procura espontânea pelos serviços de saúde. Neste último caso, substancial proporção das mulheres não é rastreada ou não cumpre as recomendações recebidas. Muitas faltam ou retardam o comparecimento às etapas seguintes do rastreamento. Estudos recentes apontam a mesma efetividade em ambos os programas quando se atingem altas taxas de prevalência de utilização da mamografia no modelo oportunístico ${ }^{15-18}$.

No Brasil, surgem atualmente dados iniciais a respeito da utilização oportunística da mamografia ${ }^{19-25}$. Evidenciamse diferenças entre as prevalências de utilização observadas nos setores públicos e privados. No entanto, o cumprimento das diretrizes do rastreamento mamográfico carece de detalhamento em ambos os segmentos de assistência à saúde. Este trabalho pretende delinear e esclarecer as características da adesão ao rastreamento mamográfico oportunístico em serviços de saúde públicos e privados.

\section{Métodos}

Trata-se de estudo longitudinal, descritivo e comparativo entre duas coortes de mulheres, identificadas em 2003 e acompanhadas durante 60 meses, após a realização de mamografia-índice em serviços de saúde públicos e privados do município de Taubaté, São Paulo, Brasil.

Entre fevereiro e julho de 2003, foram entrevistadas no momento da realização de mamografia-índice 643 mulheres, das quais 472 foram atendidas em serviços de saúde públicos e 171 em Serviços privados. Durante 60 meses, o comparecimento dos sujeitos para a realização de mamografia foi sequencialmente registrado. Não foram feitas quaisquer intervenções que motivassem maior adesão dos sujeitos às recomendações do rastreamento do câncer de mama, garantindo, assim, isenção dos pesquisadores e o caráter espontâneo e oportunístico da realização dos exames.

Da amostra inicial foram excluídas as mulheres que referiram ser portadoras de câncer mamário (7), as falecidas durante o período da pesquisa (3), as residentes fora do município de Taubaté (32) e aquelas submetidas somente a mamografia-índice ou quando o intervalo entre a realização da última mamografia e o encerramento da pesquisa foi superior a 30 meses e não foram localizadas pelo contato telefônico pessoal (141). A amostra final foi composta por 327 mulheres entrevistadas nos Serviços públicos de Saúde e outras 133 nos privados, totalizando 460 sujeitos.

O tamanho final da amostra foi validado pela Pesquisa Mundial de Saúde ${ }^{21}$, realizada no Brasil pela Organização Mundial de Saúde (OMS), também em 2003. Nos municípios com a mesma população da área desse estudo, entre 
50 e 400 mil habitantes, foram entrevistadas 253 mulheres com idade superior a 40 anos, questionadas a respeito do acesso aos exames de mamografia nos últimos três anos. A proporção de mulheres atendidas nos serviços de saúde privados $(26,5 \%)$ determinou amostras de 67 e 186 entrevistadas, respectivamente nos setores privados e públicos.

O desfecho analisado (variável dependente) foi o intervalo de repetição entre as mamografias durante os 60 meses de acompanhamento das coortes. A repetição dos exames, respeitando o intervalo de 24 meses, foi definida como critério para classificar os sujeitos em aderentes ou não aderentes ao rastreamento mamográfico. Desta forma, evitamos conflitos entre as recomendações oficiais e as privadas, anteriormente descritas como divergentes.

Foi calculada a proporção de entrevistadas que repetiu a mamografia nos primeiros 24 meses após a mamografiaíndice (intervalo índice) e, destas, a percentagem que refez o procedimento no intervalo seguinte, entre $25 \mathrm{e}$ 48 meses após o início da pesquisa. Foram feitas análises da prevalência cumulativa de repetição do sexto ao vigésimo quarto mês após a mamografia-índice. Finalmente, calculou-se a percentagem de entrevistadas que, durante os 60 meses de duração da pesquisa, realizou todos os exames em intervalos máximos de 24 meses.

As variáveis independentes relacionaram-se com características sociodemográficas da população estudada, com a modalidade de acesso aos serviços de saúde, pública ou privada, e com a frequência de utilização dos mesmos. Classificou-se como frequência regular a consulta médica e a coleta de colpocitologia oncológica realizadas a cada dois anos. Foi dada ênfase ao rastreamento mamográfico prévio e ao conhecimento das recomendações oficiais para o rastreamento mamográfico. Essas variáveis foram codificadas no menor número de estratos possíveis para facilitar a análise estatística descritiva e por meio de regressão logística.

A associação do desfecho analisado com as variáveis independentes foi estudada pela obtenção dos riscos relativos (RR), seus respectivos intervalos de confiança de 95\% (IC95\%) e do valor de p calculado pelo teste do $\chi^{2}$ e pelo teste exato de Fisher. O cálculo das razões de prevalência ajustadas foi realizado pela técnica de regressão de COX. Adotou-se $\alpha=5 \%$ como nível de significância estatística. A análise foi realizada com os softwares SAS, versão 9.1.3, e Epi-Info, versão 3.4.3.

A descrição dos dados como prevalências de realização dos exames, segundo variáveis selecionadas, em vez de proporções de cobertura associadas a expectativas de impacto de programas de saúde, baseia-se em outros estudos ${ }^{23}$ e demonstra cuidado em não confundir o significado dos resultados obtidos.

Este estudo foi previamente aprovado pelos Comitês de Ética em Pesquisa da Universidade de Taubaté (CEP/
UNITAU 0307/07) e da Faculdade de Ciências Médicas da Universidade Estadual de Campinas (CEP/UNICAMP 521/2002). Os sujeitos que participaram da pesquisa assinaram Termo de Consentimento Livre e Esclarecido, autorizando a coleta de dados proposta pelos pesquisadores e a divulgação de seus resultados.

\section{Resultados}

Das 460 entrevistadas, 71,1\% foram atendidas em Serviços públicos de Saúde e 28,9\% tinham cobertura de planos de saúde privados. As mulheres atendidas no segmento público de saúde apresentavam menor escolaridade e renda familiar $(\mathrm{p}<0,05)$. A média de idade das mulheres não diferiu entre os grupos analisados, correspondendo nos Serviços públicos e privados respectivamente a

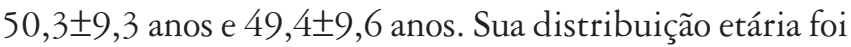
similar $(\mathrm{p}>0,05)$ e pouco mais da metade delas $(53,4 \%)$ apresentava idade inferior a 50 anos. As mulheres com mais de 60 anos de idade constituíram cerca de $14 \%$ da totalidade da amostra estudada.

Muito embora a ampla maioria das mulheres (>70\%) do total da amostra estudada frequentasse regularmente a consulta ginecológica, mais de um quarto das usuárias dos serviços públicos de saúde compareciam esporadicamente ao Serviço médico especializado e poucas $(35,3 \%)$ receberam informações a respeito da mamografia e das recomendações sobre o rastreamento mamográfico periódico. Entre aquelas que dispunham de planos de saúde privados, a proporção de consultas ginecológicas esporádicas era significativamente menor $(10,5 \%)$ e a grande maioria $(74,1 \%)$ recebeu algum tipo de informação sobre o exame $(\mathrm{p}<0,05)$.

Houve similaridade de proporções quando questionadas a respeito de antecedentes familiares de câncer de mama e presença de doenças ou anormalidades mamárias, respectivamente, 12 e $15 \%$ das entrevistadas em cada grupo estudado. Pequena percentagem das mulheres (não mais que $5 \%$ da amostra) fazia acompanhamento com mastologista ou oncologista.

Das entrevistadas, $68 \%$ referiram rastreamento mamográfico prévio nos últimos dois anos anteriores à pesquisa, porém maior proporção $(84,2 \%)$ foi registrada entre aquelas que tinham cobertura de planos de saúde privados. Negavam realização prévia de mamografia 147 mulheres (32\% do total de entrevistadas). A maior proporção $(85,7$ versus $14,3 \% ; \mathrm{p}<0,05)$, inversamente, era encontrada entre as atendidas por meio do sistema público de saúde. A média de idade das mulheres não rastreadas previamente foi cerca de sete anos mais elevada no setor público, porém não ultrapassou 50 anos de idade.

A Figura 1 reflete as prevalências cumulativas de repetição da mamografia entre o sexto e o vigésimo quarto mês após o registro do exame inicial (mamografia-índice). 


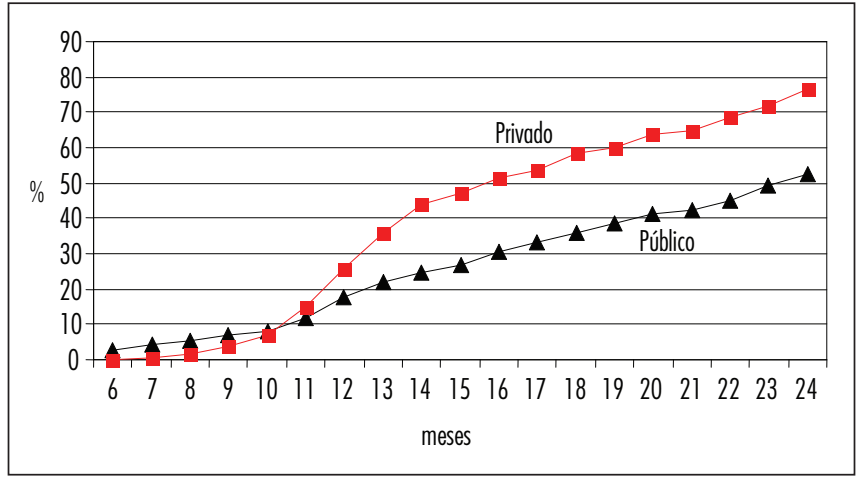

Figura 1 - Prevalência cumulativa de repetição de mamografia após exame inicial.

Tabela 1 - Percentual de adesão de acordo com intervalos de tempo, faixa etária e serviços de saúde utilizados

\begin{tabular}{|c|c|c|c|c|}
\hline & Público ( $n=327)$ & Privado ( $n=133$ ) & RR & IC95\% \\
\hline \multicolumn{5}{|l|}{ Intervalos de tempo } \\
\hline Até 24 meses & 48 & 72,2 & 0,66 & $0,57-0,77$ \\
\hline $\begin{array}{l}\text { Entre } 25 \text { e } 48 \\
\text { meses }\end{array}$ & 31,5 & 51,1 & 0,61 & $0,48-0,77$ \\
\hline Durante 60 meses & 24,5 & 42,9 & 1,75 & $1,26-2,48$ \\
\hline \multicolumn{5}{|l|}{ Faixa etária } \\
\hline Até 49 & 21,6 & 36 & 1,22 & $1,01-1,47$ \\
\hline 50 a 59 & 26,5 & 51,4 & 1,5 & $1,06-2,14$ \\
\hline 60 ou mais & 30,2 & 52,4 & 1,46 & $0,89-2,39$ \\
\hline
\end{tabular}

$\mathrm{p}<0,05$ (teste exato de Fisher), exceto acima de 60 anos.
A Tabela 1 demonstra proporções decrescentes de adesão ao rastreamento mamográfico no decorrer da pesquisa muito mais evidente entre as entrevistadas dos serviços públicos de saúde que dos privados. Ao término da pesquisa, a aderência foi mantida abaixo da metade da amostra estudada, porém foi significativamente inferior no serviço público $(24,4 \%)$ do que no privado $(42,8 \%)$.

A adesão também foi analisada de acordo com a faixa etária das pacientes (Tabela 1). Em todas as faixas etárias estudadas, as taxas de adesão foram superiores nas usuárias dos Serviços privados de Saúde. Após os 50 anos de idade, nota-se que, nos Serviços privados, as taxas de repetição ultrapassam discretamente metade da amostra estudada. Nos Serviços públicos, as taxas de prevalência de repetição mantêm-se baixas $(24,5 \%)$ independentemente da faixa etária, diferindo dos $42,9 \%$ de adesão observada entre as usuárias do setor privado $(\mathrm{RR}=1,75$; IC95\% $=1,26-2,48$ ).

Baseados no critério estabelecido de adesão adequada como a repetição da mamografia em intervalos máximos de 24 meses e a associação desse desfecho com as variáveis independentes selecionadas, calcularam-se os riscos relativos brutos $\left(R_{b r}\right)$ e ajustados pela idade das entrevistadas $\left(R_{\mathrm{aj}}\right)$ em virtude do desenho longitudinal do estudo e seus respectivos intervalos de confiança de 95\% (IC95\%). A Tabela 2 demonstra as variáveis cuja

Tabela 2 - Avaliação percentual das variáveis associadas com a adesão ao rastreamento mamográfico

\begin{tabular}{|c|c|c|c|c|c|}
\hline & & & Adesão & & \\
\hline & Presente $(n=137)$ & Ausente $(n=323)$ & $\mathbf{R R}_{b r}$ & $\mathbf{R R}_{\mathrm{oi}}$ & IC95\% \\
\hline Atendimento & & & & & \\
\hline Público & 24,5 & 75,5 & & & \\
\hline Privado & 42,9 & 57,1 & 1,75 & 1,77 & $1,26-2,48$ \\
\hline Consultas gine & & & & & \\
\hline Esporádicas & 15,9 & 84,1 & & & \\
\hline Regulares & 33,1 & 66,9 & 2,08 & 2,12 & $1,22-3,69$ \\
\hline Coleta regular & & & & & \\
\hline Não & 14,1 & 85,9 & & & \\
\hline Sim & 33,7 & 66,3 & 2,38 & 2,44 & $1,38-3,94$ \\
\hline Rastreamento & & & & & \\
\hline Não & 12,2 & 87,8 & & & \\
\hline Sim & 38 & 62 & 1,41 & 3,07 & $1,86-5,08$ \\
\hline $\begin{array}{l}\text { Recebeu infor } \\
\text { rastreamento }\end{array}$ & & & & & \\
\hline Não & 25,9 & 74,1 & & & \\
\hline Sim & 35,7 & 64,3 & 1,38 & 1,36 & $0,97-1,91$ \\
\hline $\begin{array}{l}\text { Acompanhame } \\
\text { oncologista }\end{array}$ & & & & & \\
\hline Não & 28,3 & 71,7 & & & \\
\hline Sim & 59,1 & 40,9 & 2,09 & 2,14 & $1,21-3,79$ \\
\hline Menopausa & & & & & \\
\hline Não & 22,1 & 77,9 & & & \\
\hline Sim & 37,8 & 62,2 & 1,71 & 1,95 & $1,21-3,12$ \\
\hline
\end{tabular}

$\mathrm{RR}_{\mathrm{br}}$ : risco relativo bruto; $\mathrm{RR}_{\mathrm{ai}}$ e IC95\%: risco relativo e intervalo de confiança ajustados pela idade das entrevistadas. 
associação com a adesão ao rastreamento mamográfico mostrou-se positiva.

As demais varáveis analisadas como a escolaridade, o estado marital, a ocupação profissional, a renda familiar, a presença de doenças mamárias e antecedentes familiares de câncer de mama não apresentaram associação com a adesão ao rastreamento mamográfico. A realização da mamografiaíndice somente foi observada em pequena percentagem da amostra (7,8\%), porém prevaleceu entre as usuárias dos serviços de saúde públicos $(77,8$ versus $22,2 \%)$.

\section{Discussão}

As estimativas relativas ao câncer mamário no Brasil apresentam tendências de incremento tanto de incidência quanto de mortalidade ${ }^{2,3}$. Os custos sociais e econômicos com o tratamento da doença e a perda prematura de anos de vida de mulheres jovens e produtivas justificam a inclusão deste tema em pesquisas e entre as preocupações dos gestores dos sistemas de saúde. O diagnóstico tardio da doença em nosso meio, regra comum há pouco tempo, altera-se lentamente, influenciado por propaganda maciça estimulando o autoexame, o exame clínico das mamas e a recomendação da mamografia ${ }^{5-7}$. As campanhas de estímulo ao aleitamento natural e à detecção precoce do câncer de mama ocupam espaço semelhante na mídia nacional.

Os programas de rastreamento do câncer de mama foram organizados pelos sistemas de saúde dos Estados Unidos e da Europa Ocidental ao longo das últimas cinco décadas, mesmo com protocolos diversificados e desempenho abaixo do esperado. Estão fundamentados no uso anual ou bienal da mamografia a partir de 40 ou 50 anos de idade ${ }^{10,11}$. Muitos serviços de saúde desses países exercem vigilância das recomendações propostas e convocam periodicamente as mulheres inscritas ${ }^{26,27}$, e outros, com caráter oportunístico, apresentam elevadas taxas de adesão da população ${ }^{16,17}$. São obtidos atualmente nesses países resultados animadores refletidos na diminuição das taxas de mortalidade decorrentes dessa doença ${ }^{9,18,26}$.

Surgem no Brasil, protocolos de programas regionais, como o proposto em Porto Alegre, no Rio Grande do Sul, cujo foco é baseado na vigilância ativa das mulheres inscritas em todas as etapas do rastreamento ${ }^{28}$. De modo geral, esta não é a política vigente no país. O programa para o rastreamento do câncer mamário tem caráter oportunístico. Somente a procura espontânea por qualquer consulta médica motiva a realização do exame clínico das mamas e a solicitação da mamografia. Ações mais amplas, como os mutirões para realização de mamografia, são variantes dessa mesma política de rastreamento. A vigilância das mulheres previamente rastreadas e sua convocação para cumprirem as normas recomendadas de rastreio mamográfico não têm representatividade nos programas instituídos até o presente momento, em serviços de saúde públicos ou privados ${ }^{29}$.

A avaliação das taxas de adesão é bastante controversa na literatura e depende do critério estabelecido. O questionamento a respeito da realização prévia da mamografia em períodos de dois ou três anos anteriores às pesquisas, objeto de muitos inquéritos populacionais ${ }^{19-23,25}$, não permite avaliação das taxas de adesão - apresenta vieses metodológicos e de memória - e falseia a interpretação da cobertura alcançada pelos programas de rastreamento oportunístico ${ }^{30}$.

Pelo menos dois critérios foram encontrados, em revisões recentes, que definem a aderência correta às recomendações propostas e permitem comparar resultados dos diversos estudos realizados: dois ou mais exames consecutivos respeitando o intervalo proposto entre os mesmos, isto é, repetição em no máximo 24 meses e número de exames sucessivos em determinado período de tempo de acompanhamento ${ }^{31,32}$. No presente estudo, utilizamos tempo de observação de cinco anos e definimos como aderentes ao rastreamento mamográfico as entrevistadas que repetiram todas as suas mamografias em intervalos máximos de até 24 meses. A avaliação do intervalo índice demonstra taxas bastante satisfatórias de adesão inicial e frequências cumulativas de repetição da mamografia. Cerca de metade das entrevistadas, mesmo nos serviços públicos de saúde (48\%), repetiu o exame nos primeiros 24 meses após a mamografia-índice.

O vínculo das entrevistadas com planos de saúde privados, como apontado em outros estudos, promove maior adesão e retorno mais breve para repetição da mamografia, com orientações para realização do exame em intervalos mais curtos ${ }^{33,34}$, em virtude de facilidades de acesso aos serviços médicos. Demonstramos que as curvas de frequência cumulativa de repetição da mamografia dissociam-se após os primeiros 12 meses de acompanhamento. Vinte e quatro meses após a realização da mamografia - índice, $70 \%$ das entrevistadas nos serviços de saúde privados retornaram para a segunda etapa do rastreamento mamográfico. Provavelmente, fatores sociodemográficos apontados anteriormente, como renda familiar, vínculo empregatício e escolaridade associam-se ao tipo de acesso aos serviços de saúde e contribuem definitivamente para a adesão ao rastreamento mamográfico ${ }^{22,23}$.

Vários estudos têm demonstrado que as taxas de adesão diminuem progressivamente com a extensão do tempo de acompanhamento ${ }^{31,32}$. Confirmamos tais observações, pois também, na etapa seguinte, entre 25 e 48 meses após o início da pesquisa, registramos taxas decrescentes de adesão.

$\mathrm{Na}$ segunda etapa de avaliação, o tipo de acesso aos serviços de saúde e seus fatores sociodemográficos associados provocaram disparidades pronunciadas na aderência 
ao rastreamento mamográfico. Mantiveram-se aderentes, respectivamente, $31,5 \%$ das entrevistadas nos Serviços públicos e 51,1\% nos Serviços privados, na segunda etapa da avaliação. Somente um quarto $(24,4 \%)$ das mulheres entrevistadas nos serviços de saúde públicos completou os 60 meses de acompanhamento dessa pesquisa como aderentes às normas de rastreamento mamográfico sugerido. Isto representa a falta de vigilância sobre as etapas seguintes e também ausência de convocação das mulheres, características próprias do rastreamento oportunístico.

A recomendação médica ocupa relevância indiscutível entre os fatores preditores associados à aderência ao rastreamento ${ }^{33}$. O contato com o médico, neste caso o ginecologista ou o mastologista, e outros indicadores de consulta ginecológica regular, como a coleta de colpocitologia oncológica (exame de Papanicolaou), são as variáveis fortemente associadas à realização da mamografia. Nossas análises também confirmam essas associações. No entanto, o decréscimo das taxas de adesão, observadas no decorrer da pesquisa, devem refletir o caráter oportunístico do rastreamento mamográfico vigente no Brasil, até mesmo no setor privado de assistência médica. Provavelmente, em cada etapa do rastreamento oportunístico, perde-se a chance de reforçar orientações motivadoras de crescente adesão ao rastreamento mamográfico periódico.

O documento de consenso para o controle do câncer de mama no Brasil ${ }^{12}$ propõe a mamografia bienal a partir de 50 anos de idade. Isso nos preocupa, pois, nessa faixa etária, a partir dos dados apresentados, não identificamos qualquer incremento nas taxas de adesão ao rastreamento mamográfico nos serviços de saúde públicos. As mais idosas provavelmente não comparecem aos Serviços de atendimento médico e, assim, não são alcançadas pelas recomendações dirigidas principalmente para sua faixa etária. Formas mais eficazes de estímulo ao ingresso das mulheres nos programas rastreamento mamográfico deverão ser propostas.

Atenção especial deve ser dada para as mulheres sem rastreamento prévio. Este foi um dos fatores associados ao insucesso da adesão ao rastreamento mamográfico $^{33}$. Novamente, o caráter oportunístico e a falta de informações relevantes no momento de ingresso da mulher no programa de rastreamento mamográfico não ressaltam a importância do acompanhamento periódico a partir daquele momento. Excluímos, para mantermos a veracidade dos nossos dados, a proporção de mulheres submetidas somente à mamografia-índice e com as quais não conseguimos contato posterior. Acreditamos que em condições corriqueiras da prática clínica essa proporção poderá assumir valores muito maiores.

Apesar da discussão a respeito da eficácia da mamografia, mantida incessantemente nas últimas décadas, os programas de rastreamento devem estimular o início do rastreamento mamográfico em idade apropriada e promover medidas que estabeleçam taxas crescentes de adesão e repetição dos exames. Atingir e manter altas taxas de adesão que representam obediência aos intervalos de repetição entre os exames, adequadamente definidos como inferiores a 24 meses $^{10-13}$, é crucial para se alcançar a redução dos índices de mortalidade verificados em nosso país.

\section{Agradecimentos}

A Sirlei Siani Morais e José Vilton Costa pelo tratamento estatístico dos dados desse trabalho. A Lúcio Tito Gurgel pela revisão das tabelas apresentadas.

\section{Referências}

1. Jemal A, Siegel R, Ward E, Hao Y, Xu J, Thun M. Cancer statistics, 2009. CA Cancer J Clin. 2009;59(4):225-49.

2. Brasil. Ministério da Saúde. Instituto Nacional do Câncer [Internet]. Estimativas 2010: incidência de câncer no Brasil. Rio de Janeiro; 2009 [citado 2010 Jan 9]. Disponível em: <http://www.inca. gov.br/estimativa/2010/estimativa20091201.pdf >

3. Brasil. Ministério da Saúde. Instituto Nacional do Câncer [Internet]. Atlas de mortalidade por câncer. Rio de Janeiro; 1996-2009 [citado 2009 Set 27]. Disponível em: < http://mortalidade. inca.gov.br/>

4. Rosenberg J, Chia YL, Plevrits S. The effect of age, race, tumor size, tumor grade, and disease stage on invasive ductal breast cancer survival in the U.S. SEER database. Breast Cancer Res Treat. $2005 ; 89(1): 47-54$.
5. São Paulo (Estado). Secretaria Estadual da Saúde. Fundação Oncocentro de São Paulo [Internet]. Boletim de Registro Hospitalar de Câncer. São Paulo; 2003 [citado 2009 Maio 30]. Disponível em: <http://www.fosp.saude.sp.gov.br/docs/boletins/rhc18. pdf >

6. Thuler LCS, Mendonça GA. Estadiamento inicial dos casos de câncer de mama e colo do útero em mulheres brasileiras. Rev Bras Ginecol Obstet. 2005;27(1 1):656-60.

7. Martins E, Freitas-Junior R, Curado MP, Freitas NMA, Oliveira $J C$, et al. Evolução temporal dos estádios do câncer de mama ao diagnóstico em um registro de base populacional no Brasil Central. Rev Bras Ginecol Obstet. 2009;31 (5):219-23.

8. Keen JD, Keen JE. What is the point: will screening mammography save my life? BMC Med Inform Decis Mak. 2009;9:18. 
9. Tabar L, Yen MF, Vitak B, Chen HH, Smith RA, Duffy SW. Mammography service screening and mortality in breast cancer patients: 20-year follow-up before and after introduction of screening. Lancet. 2003;361 (9367):1405-10.

10. US Preventive Services Task Force. Screening for breast cancer: U.S. Preventive Services Task Force recommendation statement. Ann Intern Med. 2009;151(10):716-26.

11. Smith RA, Cokkinides V, Brawley OW. Cancer screening in the United States, 2009: a review of current American Cancer Society guidelines and issues in cancer screening. CA Cancer J Clin. 2009;59(1):27-41.

12. Brasil. Ministério da Saúde. Instituto Nacional de Câncer. Controle do câncer de mama: documento de consenso [Internet]. Rio de Janeiro; 2004 [citado 2007 Mai 4]. Disponível em: <http://www. inca.gov.br/publicacoes/Consensointegra.pdf>

13. Kemp C, Petti DA, Ferraro O, Elias S. Câncer de mama: prevenção secundária [Internet]. Brasília (DF): Associação Médica Brasileira e Conselho Federal de Medicina; 2002 [citado 2009 Mai 4]. Disponível em: <http://www.projetodiretrizes.org.br/projeto_ diretrizes/026.pdf>

14. Lui CY, Lam HS, Chan LK, Tam KF, Chan CM, Leung TY, et al. Opportunistic breast cancer screening in Hong Kong; a revisit of the Kwong Wah Hospital experience. Hong Kong Med J. 2007;13(2): 106-13.

15. Hertl K, Primic-Zakeli M, Zgajnar J, Kocijancic I. Performance of opportunistic breast cancer screening in Slovenia. Neoplasma. 2006;53(3):237-41.

16. Bulliard JL, Ducros C, Jemelin C, Arzel B, Fioretta G, Levi F. Effectiveness of organised versus opportunistic mammography screening. Ann Oncol. 2009;20(7): 1 199-202.

17. de Gelder R, Bulliard JL, de Wolf C, Fracheboud J, Draisma $G$, Schopper D, et al. Cost-effectiveness of opportunistic versus organised mammography screening in Switzerland. Eur J Cancer. 2009;45(1):127-38.

18. Schopper D, de Wolf $C$. How effective are breast cancer screening programmes by mammography? Review of the current evidence. Eur J Cancer. 2009;45(1 1):1916-23.

19. Brasil. Ministério da Saúde. Instituto Nacional de Câncer. Inquérito domiciliar sobre comportamento de risco e morbidade referida de doenças e agravos não transmissíveis [Internet]. Rio de Janeiro; 2003 [citado em 2007 Mai 15]. Disponível em: <http://www. inca.gov.br/publicacoes/publicacao_inquerito22_06.pdf $\geq$

20. Brasil. Instituto Brasileiro de Geografia e Estatística (IBGE). Pesquisa Nacional por Amostras de Domicílios: acesso e utilização de serviços de saúde. Rio de janeiro: IBGE; 2005.

21. Szwarcwald CL, Viacava F, Vasconcellos MTL, Leal MC, Azevedo LO, Queiroz RSB, et al. Pesquisa Mundial de Saúde 2003: o Brasil em números. RADIS/FIOCRUZ. 2004;23:14-33.
22. Leal MC, Gama SGN, Frias P, Szwarcwald CL. Healthy lifestyles and access to periodic health exams among Brazilian women. Cad. saúde pública. 2005;21(Supl 1):S78-S88.

23. Novaes $H M D$, Braga $P E$, Schout $D$. Fatores associados à realização de exames preventivos para câncer nas mulheres brasileiras, PNAD 2003. Cienc. saude coletiva. 2006;1 1 (4):1023-35

24. Marchi AA, Gurgel MSC, Fonsechi-Carvasan G. Rastreamento mamográfico do câncer de mama em serviços de saúde públicos e privados. Rev Bras Ginecol Obstet. 2006;28(4):214-9.

25. Brasil. Ministério da Saúde. Secretaria de Vigilância em Saúde [Internet]. VIGITEL Brasil 2007: Vigilância de fatores de risco e proteção para doenças crônicas por inquérito telefônico. Brasília (DF); 2008 [citado 2010 Jan 10]. Disponível em: < http://portal.saude.gov.br/portal/arquivos/pdf/vigitel2007_ final_web.pdf>

26. Lynge E, Olsen AH, Fracheboud J, Patnick J. Reporting of performance indicators of mammography screening in Europe. Eur J Cancer Prev. 2003:12(3):213-22.

27. Coburn NG, Chung MA, Fulton J, Cady B. Decreased breast cancer tumor size, stage, and mortality in Rhode Island: an example of a well-screened population. Cancer Control. 2004;11(4): 222-230.

28. Caleffi M, Ribeiro RA, Duarte Filho DL, Ashton-Prolla P, Bedin A Jr, Skonieski GP, et al. A model to optimize public health care and downstage breast cancer in limited-resource populations in southern Brazil. (Porto Alegre Breast Health Intervention Cohort). BMC Public Health. 2009;9:83.

29. Brasil. Ministério da Saúde. Instituto Nacional de Câncer [Internet]. Programa Nacional de Controle do Câncer do Colo de Útero e Mama - Viva Mulher. Rio de Janeiro; 2005 [citado 2007 Maio 15]. Disponível em: <http://www.inca.gov.br/conteudo_view. asp? $\mathrm{id}=140>$

30. Viacava F, Souza-Junior PRB, Moreira RS. Estimativas da cobertura de mamografia segundo inquéritos de saúde no Brasil. Rev Saúde Pública. 2009;43(Supl 2):117-25.

31. Clark MA, Rakowski W, Bonacore LB. Repeat mammography: prevalence estimates and considerations for assessment. Ann Behav Med. 2003;26(3):201-11.

32. Boudreau DM, Luce $C L$, Ludman $E$, Bonomi AE, Fishman PA. Concordance of population-based estimates of mammography screening. Prev Med. 2007;45(4):262-6.

33. Barr JK, Franks AL, Lee NC, Herther P, Schachter M. Factors associated with continued participation in mammography screening. Prev Med. 2001;33(6):661-7.

34. Bobo JK, Shapiro JA, Schulman J, Wolters CL. On-schedule mammography rescreening in the National Breast and Cervical Cancer Early Detection Program. Cancer Epidemiol Biomarkers Prev. 2004;13(4):620-30. 